\title{
ПРИМЕНЕНИЕ МЕТОДА ИНСТРУМЕНТАЛЬНЫХ ПЕРЕМЕННЫХ ДЛЯ ПАРАМЕТРИЧЕСКОЙ ИДЕНТИФИКАЦИИ РАСПРЕДЕЛЕННОЙ ДИНАМИЧЕСКОЙ СИСТЕМЫ
}

\author{
А. В. Копытин, Е. А. Копытина \\ Воронежский государственный университет
}

Поступила в редакцию 22.11.2018 г.

\begin{abstract}
Аннотация. Предложен вариант метода инструментальных переменных для параметрической идентификации уравнений математической физики, описывающих динамику пространственно-распределенных процессов, на основе экспериментальных многомерных временных рядов. Проведенный вычислительный эксперимент показывает значительное улучшение качества оценок параметров уравнения по сравнению с методом наименьших квадратов.

Ключевые слова: уравнения в частных производных; параметрическая идентификация; многомерная авторегрессия; МНК; метод инструментальных переменных.

Annotation. A variant of the method of instrumental variables for the parametric identification of equations of mathematical physics describing the dynamics of spatially-distributed processes on the basis of experimental multidimensional time series is proposed. The computational experiment performed shows a significant improvement in the quality of estimates of the parameters of the equation in comparison with the ordinary least squares method.

Keywords: partial differential equations; parametric identification; multidimensional autoregression; OLS; instrumental variables method.
\end{abstract}

\section{ВВЕДЕНИЕ}

Хорошо известна проблема параметрической идентификации моделей динамических систем. Исследованию этой проблемы посвящено много работ (см., например., [1-10]). При этом использование известных подходов ограничено рядом условий: часть подходов основана на обработке статистики, полученной в результате активного эксперимента, который невозможно провести для некоторых объектов исследования. Многие работы ограничены рамками моделей, описываемых обыкновенными дифференциальными уравнениями.

Продолжая исследования, начатые в [1113], будем рассматривать широкий класс пространственно-распределенных динамических систем, для которых характерны диффузионные процессы, процессы адвекции или их сочетание. Соответствующее дифферен-

(c) Копытин А. В., Копытина Е. А., 2018 циальное уравнение в частных производных с начальным и граничными условиями имеет следующий общий вид:

$$
\begin{gathered}
\frac{\partial y}{\partial t}+v \frac{\partial y}{\partial l}=D \frac{\partial^{2} y}{\partial l^{2}}, \\
y(0, l)=\phi(l), \\
y\left(t, l^{\min }\right)=f_{1}(t), \quad y\left(t, l^{\max }\right)=f_{2}(t),
\end{gathered}
$$

где $v$ - скорость адвекции, $D$ - коэффициент диффузии, $l$ - пространственная координата.

Источником информации о поведении системы являются данные натурных измерений переменной $y_{i}^{k}$ с погрешностью $\varepsilon_{i}^{k}$ в виде «белого шума» $-x_{i}^{k}=y_{i}^{k}+\varepsilon_{i}^{k}$ в последовательные моменты времени $\left\{t_{k}\right\}_{k=0}^{n}$ в узлах одномерной пространственной регулярной сетки $\left\{l_{i}\right\}_{i=0}^{m}$, т. е. многомерный временной ряд. Рассмотрение одномерной сетки ничем не ограничивает дальнейшие исследования, зато позволяет избежать громоздких построений, характерных для плоских и объемных пространств.

Задача заключается в верификации процессов конвективной диффузии на основе 


\section{A. В. Копьтин, E. А. Копьтина}

анализа многомерных временных рядов и разработке алгоритмов параметрической идентификации механистической модели с постоянными коэффициентами по наблюдаемым значениям $x_{i}^{k}$.

\section{МЕТОДЫ И МАТЕРИАЛЫ}

Для решения задачи составим явную четырехточечную разностную схему для уравнения (1):

$$
\begin{aligned}
& \frac{y_{i}^{k+1}-y_{i}^{k}}{\Delta t}+v \frac{y_{i+1}^{k}-y_{i-1}^{k}}{2 \Delta l}=D \frac{y_{i+1}^{k}-2 y_{i}^{k}+y_{i-1}^{k}}{\Delta l^{2}}, \\
& y_{i}^{k+1}=\left(b_{1}+b_{2}\right) y_{i-1}^{k}+\left(1-2 b_{2}\right) y_{i}^{k}+\left(b_{2}-b_{1}\right) y_{i-1}^{k}, \\
& \text { где } b_{1}=\frac{v \Delta t}{2 \Delta l}, b_{2}=\frac{D \Delta t}{\Delta l^{2}} .
\end{aligned}
$$

Уравнения (2) можно записать в виде

$$
y_{i}^{k+1}=a_{1} y_{i-1}^{k}+a_{2} y_{i}^{k}+a_{3} y_{i-1}^{k}, k=0,1, \ldots, n-1,
$$

где $a_{1}, a_{2}, a_{3}$ - оцениваемые регрессионные параметры, связанные с параметрами $b_{1}$ и $b_{2}$ следующими соотношениями:

$$
\left\{\begin{array}{l}
b_{1}+b_{2}=a_{1}, \\
1-2 b_{2}=a_{2}, \\
b_{2}-b_{1}=a_{3} .
\end{array}\right.
$$

Запишем уравнения (3) при $k=1, \ldots, n-1$ в матричной форме

$$
\mathbf{y}=\mathbf{Y a},
$$

где

$$
\mathbf{y}=\left(\begin{array}{c}
y_{i}^{2} \\
y_{i}^{3} \\
\vdots \\
y_{i}^{n}
\end{array}\right), \quad \mathbf{Y}=\left(\begin{array}{ccc}
y_{i-1}^{1} & y_{i}^{1} & y_{i+1}^{1} \\
y_{i-1}^{2} & y_{i}^{2} & y_{i+1}^{2} \\
\vdots & \vdots & \vdots \\
y_{i-1}^{n-1} & y_{i}^{n-1} & y_{i+1}^{n-1}
\end{array}\right), \quad \mathbf{a}=\left(\begin{array}{l}
a_{1} \\
a_{2} \\
a_{3}
\end{array}\right) .
$$

Обозначим $\mathbf{x}=\mathbf{y}+\boldsymbol{\varepsilon}, \mathbf{X}=\mathbf{Y}+\mathbf{E}$, где $\boldsymbol{\varepsilon}$ и $\mathbf{E}-$ соответственно вектор-столбец и матрица ошибок измерений:

$$
\boldsymbol{\varepsilon}=\left(\begin{array}{c}
\varepsilon_{i}^{2} \\
\varepsilon_{i}^{3} \\
\vdots \\
\varepsilon_{i}^{n}
\end{array}\right), \quad \mathbf{E}=\left(\begin{array}{ccc}
\varepsilon_{i-1}^{1} & \varepsilon_{i}^{1} & \varepsilon_{i+1}^{1} \\
\varepsilon_{i-1}^{2} & \varepsilon_{i}^{2} & \varepsilon_{i+1}^{2} \\
\vdots & \vdots & \vdots \\
\varepsilon_{i-1}^{n-1} & \varepsilon_{i}^{n-1} & \varepsilon_{i+1}^{n-1}
\end{array}\right) .
$$

В новых обозначениях уравнение (5) примет вид

$$
\mathbf{x}=\mathbf{X a}+(\boldsymbol{\varepsilon}-\mathbf{E a}) .
$$

Уравнение (6) позволяет найти обычную МНК-оценку $\mathbf{a}$ вектора параметров а:

$$
\hat{\mathbf{a}}=\left(\mathbf{X}^{T} \mathbf{X}\right)^{-1} \mathbf{X}^{T} \mathbf{x}=\mathbf{a}+\left(\mathbf{X}^{T} \mathbf{X}\right)^{-1} \mathbf{X}^{T}(\boldsymbol{\varepsilon}-\mathbf{E a}) .
$$

Найдем математическое ожидание левой и правой частей выражения (7):

$$
M(\hat{\mathbf{a}})=\mathbf{a}+M\left[\left(\mathbf{X}^{T} \mathbf{X}\right)^{-1} \mathbf{X}^{T}(\boldsymbol{\varepsilon}-\mathbf{E a})\right] .
$$

Как видно из полученного равенства (8), математическое ожидание оценки $\mathbf{a}$ отличается от истинного вектора а на величину $M\left[\left(\mathbf{X}^{T} \mathbf{X}\right)^{-1} \mathbf{X}^{T}(\boldsymbol{\varepsilon}-\mathbf{E a})\right] \neq 0$, которая интерпретируется как смещение компонент вектора оценки.

Наличие смещения может существенно повлиять на оценку параметров $b_{1}$ и $b_{2}$, определяемых на основе системы уравнений (4). Эту проблему можно уменьшить за счет использования инструментальных переменных $[14,15]$.

Метод инструментальных переменных предполагает наличие набора переменных $\mathbf{Z}$, называемых инструментами. Инструменты должны быть некоррелированны с ошибкой $\boldsymbol{\varepsilon}$ - Еа и, напротив, как можно сильнее коррелированны с регрессорами $\mathbf{X}$. Количество инструментов должно быть не меньше количества регрессоров.

Как только инструменты выбраны, можно применить двухшаговый МНК для оценивания вектора а. На первом шаге находятся обычные МНК-оценки $\hat{\mathbf{B}}=\left(\mathbf{Z}^{T} \mathbf{Z}\right)^{-1} \mathbf{Z}^{T} \mathbf{X}$ матрицы параметров В уравнения регрессии $\mathbf{X}=\mathbf{Z B}+\mathbf{V}$. В результате получаем следующие оценки исходных переменных $\mathbf{X}$ :

$$
\hat{\mathbf{X}}=\mathbf{Z} \hat{\mathbf{B}}=\mathbf{Z}\left(\mathbf{Z}^{T} \mathbf{Z}\right)^{-1} \mathbf{Z}^{T} \mathbf{X}=\mathbf{P}_{\mathbf{Z}} \mathbf{X},
$$

где $\mathbf{P}_{\mathbf{Z}}=\mathbf{Z}\left(\mathbf{Z}^{T} \mathbf{Z}\right)^{-1} \mathbf{Z}^{T}$.

На втором шаге также обычным МНК оценивается исходная модель (6) с заменой регрессоров $\mathbf{X}$ на их оценки (9), полученные на первом шаге:

$$
\hat{\mathbf{a}}_{I V}=\left(\hat{\mathbf{X}}^{T} \hat{\mathbf{X}}\right)^{-1} \hat{\mathbf{X}}^{T} \mathbf{x}=\left(\mathbf{X}^{T} \mathbf{P}_{\mathbf{Z}}^{T} \mathbf{P}_{\mathbf{Z}} \mathbf{X}\right)^{-1} \mathbf{X}^{T} \mathbf{P}_{\mathbf{Z}}^{T} \mathbf{x} .
$$

Учитывая, что $\mathbf{P}_{\mathbf{Z}}^{T}=\mathbf{P}_{\mathbf{Z}}, \mathbf{P}_{\mathbf{Z}}^{T} \mathbf{P}_{\mathbf{Z}}=\mathbf{P}_{\mathbf{Z}}$, окончательно получаем формулу оценок метода инструментальных переменных:

$$
\hat{\mathbf{a}}_{I V}=\left(\mathbf{X}^{T} \mathbf{P}_{\mathbf{Z}} \mathbf{X}\right)^{-1} \mathbf{X}^{T} \mathbf{P}_{\mathbf{Z}} \mathbf{x}, \quad \mathbf{P}_{\mathbf{Z}}=\mathbf{Z}\left(\mathbf{Z}^{T} \mathbf{Z}\right)^{-1} \mathbf{Z}^{T} \text {. }
$$

В нашем случае в качестве инструментов предлагается выбрать набор из пяти лагированных переменных: 
Применение метода инструментальных переменных для параметрической идентификации ...

$$
\mathbf{Z}=\left(\begin{array}{ccccc}
x_{i-2}^{0} & x_{i-1}^{0} & x_{i}^{0} & x_{i+1}^{0} & x_{i+2}^{0} \\
x_{i-2}^{1} & x_{i-1}^{1} & x_{i}^{1} & x_{i+1}^{1} & x_{i+2}^{1} \\
\vdots & \vdots & \vdots & \vdots & \vdots \\
x_{i-2}^{n-2} & x_{i-1}^{n-2} & x_{i}^{n-2} & x_{i+1}^{n-2} & x_{i+2}^{n-2}
\end{array}\right) .
$$

Выбранные таким образом инструменты будут с одной стороны некоррелированы с ошибкой $\boldsymbol{\varepsilon}-\mathbf{E a}$, поскольку погрешности измерений $\varepsilon_{i}^{k}$ в разные моменты времени независимы, а с другой - линейно выражать регрессоры $\mathbf{X}$.

\section{РЕЗУЛЬТАТЫ И ИХ ОБСУЖДЕНИЕ}

Для проведения исследований удобно воспользоваться данными модельного эксперимента. Для этого необходимо найти решение исходного дифференциального уравнения (1) с заданными значениями параметров $v$ и $D$, которые легко пересчитываются в параметры $b_{1}$ и $b_{2}$ разностной схемы. Затем выполнить регулярную дискретизацию полученного решения и добавить погрешность $\varepsilon$ в виде «белого шума» с различной интенсивностью. Полученные статистические данные будут использованы для получения оценок $\hat{b}_{1}$ и $b_{2}$ параметров разностной схемы. Таким образом, модельный эксперимент позволяет провести наглядное сравнение исходных значений параметров и их оценок при различных методах получения оценок и различных интенсивностях помехи.

Рассмотрим задачу (1) на отрезке $[1 ; 3]$ с такими функциями $\phi, f_{1}$ и $f_{2}$ что ее решение имеет вид:

$$
\begin{gathered}
y(t, l)= \\
=e^{\frac{v}{2 D}\left(l-\frac{v t}{2}\right)}\left(e^{-D t} \sin l+e^{-4 D t} \sin 2 l+e^{-9 D t} \sin 3 l\right) .
\end{gathered}
$$

Пусть значения параметров $v$ и $D$ равны соответственно 2 и 3; шаг по пространственной координате $\Delta l=0,1$; шаг по времени $\Delta t=\Delta l^{2} /(4 D)$, что соответствует условиям Куранта для обеспечения устойчивости аппроксимирующей разностной схемы; $n=1001$.

Далее к значениям $y_{i}^{k}$ решения (11) в узлах пространственно-временной сетки добавим смоделированную с помощью генератора случайных чисел погрешность в виде «белого шума» со стандартным отклонением $\sigma$ и по полученным значениям $x_{i}^{k}$ найдем сначала МНК-оценки $\hat{\mathbf{a}}$ параметров уравнения (6), оценки этих параметров с помощью метода инструментальных переменных и оценки $\hat{b}_{1}$ и $\hat{b}_{2}$ с помощью системы (4) и предложенной методики. Очевидно, что результаты параметрической идентификации могут существенно зависеть от интенсивности «белого шума», задаваемой стандартным отклонением случайной погрешности наблюдений $\sigma$. Для проведения эксперимента были выбраны три уровня погрешности: $\sigma=0,001 ; \sigma=0,005$; $\sigma=0,01$. Результаты идентификации представлены в табл. 1.

Данные табл. 1 показывают, что при всех трех уровнях погрешности средняя абсолютная ошибка в процентах метода инструментальных переменных значительно меньше, чем ошибка МНК, хотя и является достаточно большой.

Таблица 1

Средняя абсолютная ошибка в проиентах (МАРЕ)

\begin{tabular}{lcccc}
\hline & & \multicolumn{3}{c}{$\sigma$} \\
\cline { 3 - 5 } & & 0,001 & 0,005 & 0,01 \\
\hline МнК & $v$ & 7,934 & 97,825 & 150,964 \\
& $D$ & 1,018 & 16,222 & 26,048 \\
Метод & & & & \\
инструментальных & & & & \\
переменных & & & & \\
& $v$ & 4,864 & 25,468 & 56,656 \\
& $D$ & 0,536 & 2,697 & 6,286 \\
\hline
\end{tabular}


Средние значения оценок параметров

\begin{tabular}{lcccc}
\hline & & \multicolumn{3}{c}{$\sigma$} \\
\cline { 3 - 5 } & & 0,001 & 0,005 & 0,01 \\
\hline МНК & $v$ & 2,144 & 3,956 & 5,013 \\
& $D$ & 3,029 & 3,487 & 3,781 \\
Метод & & & & \\
инструментальных & & & & \\
переменных & & & & \\
& $v$ & 1,999 & 2,047 & 2,160 \\
& $D$ & 2,993 & 2,991 & 3,022 \\
\hline
\end{tabular}

Модельный эксперимент по каждому методу получения оценок и при каждом уровне интенсивности помехи повторялся 500 раз, что позволило получить среднеарифметические значения оценок параметров $v$ и $D$. Средние значения оценок можно рассматривать как хорошее приближение к их математическому ожиданию. В этом случае разницу между средним значением оценки и ее истинным значением можно принять за величину смещения. Результаты сравнения средних значений исследуемых оценок при различных интенсивностях помех получаются на основании данных табл. 2.

Сравнение средних значений с истинными значениями параметров показывает, что величина смещения оценок МНК существенна даже при небольших погрешностях и возрастает с ростом погрешности. При этом смещение оценок метода инструментальных переменных на порядки меньше и остается приемлемым даже при самом высоком уровне погрешности, что подтверждает целесообразность применения данного метода.

\section{ЗАКЛЮЧЕНИЕ}

Результаты проведенных экспериментов показывают, что использование для оценки параметров дифференциального уравнения (1) метода наименьших квадратов может привести к существенным искажениям истинных значений параметров в условиях высокого уровня погрешностей наблюдений за многомерными временными рядами в узлах разностной схемы.

Предложенный вариант метода инструментальных переменных, как показывают результаты табл. 1 и 2, существенно улучшает качество оценки.

\section{СПИСОК ЛИТЕРАТУРЫ}

1. A Bayesian approach to parameter estimation in HIV dynamical models / H. Putter [et al.] // Statistics in Medicine. - 2002. Vol. 21. - P. 2199-2214.

2. Huang, Y. Hierarchical Bayesian methods for estimation of parameters in a longitudinal HIV dynamic system / Y. Huang, D. Liu, H. Wu // Biometrics. - 2006. - Vol. 62. - P. 413-423.

3. Huang, Y. A Bayesian approach for estimating antiviral efficacy in HIV dynamic models / Y. Huang, H. Wu // Journal of Applied Statistics. - 2006. - Vol. 33. - P. 155-174.

4. Parameter estimation for differential equations: a generalized smoothing approach (with discussion) / J. O. Ramsay [и др.] // Journal of the Royal Statistical Society. Series B. - 2007. Vol. 69. - P. 741-796.

5. Liang, $H$. Parameter estimation for differential equation models using a framework of measurement error in regression models / H. Liang, $\mathrm{H}$. Wu // Journal of the American Statistical Association. - 2008. - Vol. 103. - P. 1570-1583.

6. Chen, J. Efficient local estimation for time-varying coefficients in deterministic dynamic models with applications to HIV-1 dynamics / 
Применение метода инструментальных переменных для параметрической идентификации ...

J. Chen, H. Wu // Journal of the American Statistical Association. - 2008. - Vol. 103. - P. 369-384.

7. Cao, J. Penalized nonlinear least squares estimation of time-varying parameters in ordinary differential equations / J. Cao, J. Z. Huang, $\mathrm{H}$. Wu // Journal of Computational and Graphical Statistics. - 2012. - Vol. 21. - P. 42-56.

8. Muller, T. Fitting parameters in partial differential equations from partially observed noisy data / T. Muller, J. Timmer // Physical Review, D. - 2002. - Vol. 171. P. 1-7.

9. Muller, T. Parameter identification techniques for partial differential equations / T. Muller, J. Timmer // International Journal of Bifurcation and Chaos. - 2004. - Vol. 14. - P. 2053-2060.

10. Parameter estimation of partial differential equation models / X. Xun [et al.] // Journal of the American Statistical Association. - 2013. Vol. 108. - P. 1009-1020.

11. Modeling of nonstationary distributed processes on the basis of multidimensional time

Копытин А. В. - к. ф.-м. н., доцент кафедры информационных технологий управления, факультет компьютерных наук, Воронежский государственный университет.

E-mail: alexkopytin@gmail.com

Копытина Е. А. - ассистент кафедры информационных технологий управления, факультет компьютерных наук, Воронежский государственный университет.

E-mail: zhemkaterina@yandex.ru series / M. G. Matveev [et al.] // Procedia Engineering. - 2017. - Vol. 201. - P. 511-516.

12. Verification of the convective diffusion process based on the analysis of multidimensional time series / M. G. Matveev [et al.] // CEUR Workshop Proceedings. - 2017. - Vol. 2022. P. 354-358.

13. Копьтин, А. В. Применение расширенного фильтра Калмана для идентификации параметров распределенной динамической системы / А. В. Копытин, Е. А. Копытина, М. Г. Матвеев // Вестник Воронеж. гос. ун-та. Сер. Системный анализ и информационные технологии. - Воронеж, 2018. - № 3. - С. 44-50.

14. Bowden, R. J. Instrumental variables / R. J. Bowden, D. A. Turkington. - New York: Cambridge University Press, 1984. - 227 p.

15. White, H. Asymptotic theory for econometricians / H. White. - New York : Academic Press, 2001. - 264 p.

Kopytin A. V. - Ph. D. of Physical and Mathematical Sciences, Associate Professor, Department of Information Technologies in Management, Computer Sciences Faculty, Voronezh State University. E-mail: alexkopytin@gmail.com

Kopytina E. A. - Postgraduate Student, Department of Information Technologies in Management, Computer Sciences Faculty, Voronezh State University.

E-mail: zhemkaterina@yandex.ru 